\title{
Simulation Studies of Solar Prominence Formation
}

\author{
G. S. Choe ${ }^{1}$ \\ Tongmyong University of Information Technology, Pusan 608-080, Korea \\ C. Z. Cheng \\ Princeton Plasma Physics Laboratory, Princeton University, Princeton, \\ NJ 08543, USA
}

\begin{abstract}
A series of numerical simulations are presented for formation of solar prominences. We have investigated the dynamic and thermodynamic evolution of coronal plasma in response to photospheric horizontal motions. In three different setups of field configurations and footpoint motions, different types of prominences are found to be formed by thermal instability.
\end{abstract}

\section{Introduction}

Solar prominences are always observed above polarity inversion lines and this indicates that magnetic fields play an important role in subsistence of prominences. Martin (1990) discussed the conditions for prominence formation observable in the optical wavelengths, which can be summarized by two phrases: sheared magnetic fields and converging motions toward the polarity inversion lines. Although the origin of magnetic shear is not clearly known, it can be attributed to shearing and converging motions at the field line footpoint level as observed by Rompolt and Bogdan (1986).

Based on these observations, we have investigated the dynamic and thermodynamicevolution of the coronal plasma and magnetic fields employing shearincreasing footpoint motions as boundary conditions. In our numerical models, the field-aligned heat conduction, the radiative cooling and a phenomenological form of the coronal heating are taken into account to explore the thermal instability (for details see Choe and Lee 1992, Choe 1995). Bipolar (Cases A and B) and quadrupolar (Case C)field configurations, respectively, are considered in three different cases.

\section{Case A: Formation of a Kippenhahn-Schlüter Type Prominence}

In this case, a shearing footpoint motion is applied to a single magnetic arcade. The first stage of the evolution is almost adiabatic and the temperature is decreased over the whole domain by the expansion of the arcade. The radiative

\footnotetext{
${ }^{1}$ Also at Princeton Plasma Physics Laboratory, Princeton, NJ 08543, USA
} 
cooling is gradually enhanced by the adiabatic cooling and finally a thermal instability is effected. At $t \sim 7500 \mathrm{~s}$ the temperature drops quickly to about $10^{4} \mathrm{~K}$ in a small patch and the material in the vicinity is sucked into it along field lines. Above and below this region, thermal instability takes place successively upward and downward. Consequently, the condensed material forms a sheet-like structure, in which the peak density reaches about 70 times the initial value. The field lines are pressed down by the prominence mass and form a dip structure, which is a typical signature of the Kippenhahn-Schlüter prominence model (see Figure 1). Above a certain height, the cooled material contracts at the edges of the cooled region and the condensed material slides down along field lines. This process evacuates the flux tubes and creates a coronal cavity as shown in Figure 1.

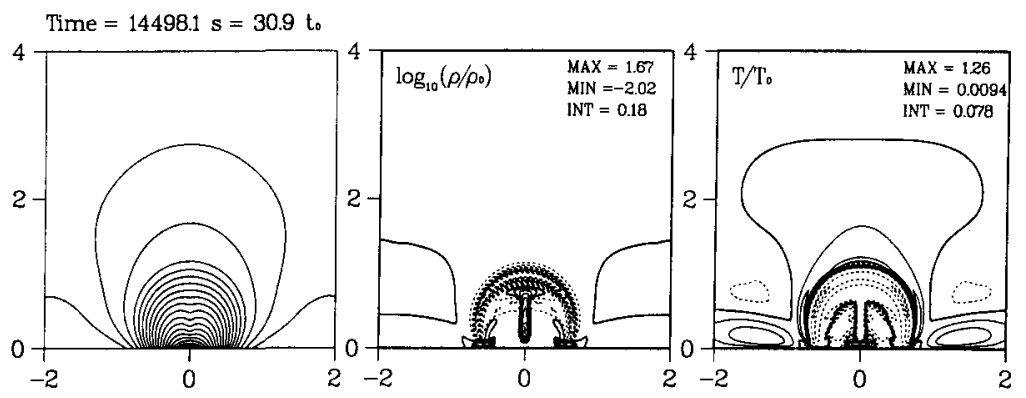

Figure 1. Case A: A Kippenhahn-Schlüter type prominence with a coronal cavity. Field lines and contours of density $\left(\log \left(\rho / \rho_{0}\right)\right)$ and temperature $\left(T / T_{0}\right)$ are shown. The dotted lines refer to decrease of the quantities from the initial values.

\section{Case B: Formation of a Kuperus-Raadu Type Prominence}

In Case $\mathbf{B}$, a converging motion toward the polarity inversion line is applied to a sheared bipolar arcade to increase the magnetic shear more effectively. In the expanding arcade, a thermal instability develops as in Case A, but the condensed material slides down along field lines because the field line curvature in the cool region is higher than that in Case A. As the footpoints converge, a current layer develops in the lower part of the arcade where magnetic reconnection takes place. The highly enhanced density in the magnetic island triggers another thermal instability and the condensed material is accumulated at the bottom of the looped field lines, finally forming a sheet-like structure. About $92 \%$ of the mass in the island is concentrated in the prominence and the rest of the island volume constitutes a low pressure cavity (see Figure 2). In Case B, an inverse polarity prominence of Kuperus-Raadu type is attained. 


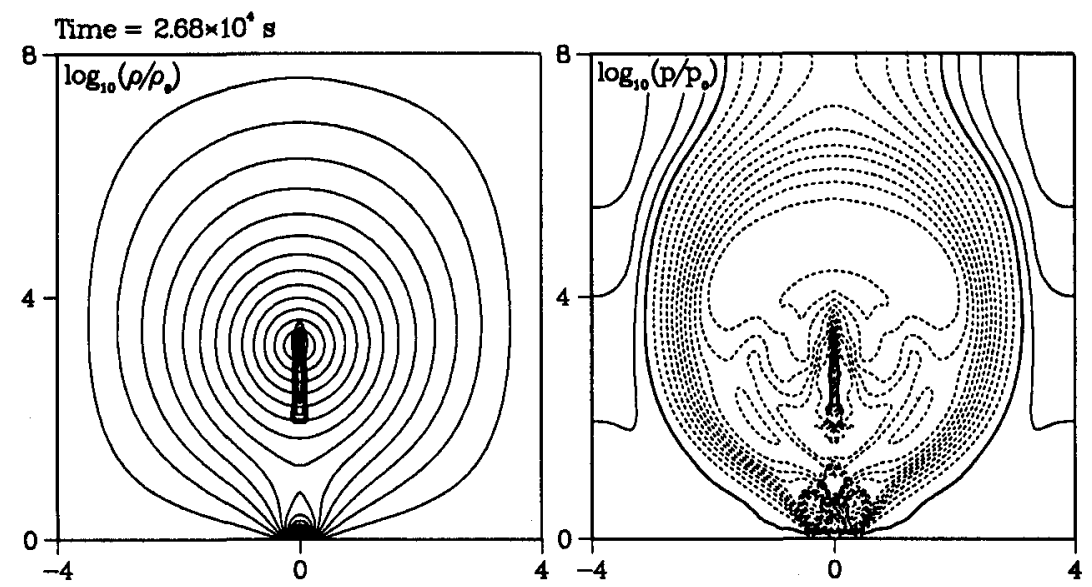

Figure 2. Case B: Density and pressure profiles of a Kuperus-Raadu type prominence. On the left, contours of $\log \left(\rho / \rho_{0}\right) \geq 0$ are superimposed on the magnetic field lines. The corresponding contours of $\log \left(p / p_{0}\right)$ are shown on the right.

\section{Case C: Prominence Formation Between Two Bipolar Regions}

In this case, two adjacent bipolar regions are mimicked by a quadrupolar field without an X-point initially. As the footpoint shear increases, both arcades individually expand and form a current sheet between them, where magnetic reconnection takes place. The expansion of arcades induce a quasiadiabatic cooling except near and below the X-line. The density is decreased within the two expanding arcades except near the $\mathrm{X}$-line, but is increased in the overlying arcade. Thus, a thermal instability takes place in the overlying arcade and the upward and downward propagation of condensation results in a vertical sheet structure of the prominence (Figure 3). Although the field lines supporting the prominence material are topologically similar to those in the KippenhahnSchlïter model, the prominence field will be observed to have an inverse polarity. Therefore, a Kippenhahn-Schlüter type cannot always be equated with a normal polarity prominence.

\section{Discussion}

In our models, prominences are formed by condensation of coronal plasma. However, we cannot exclude the possibility that the cool prominence material may be directly transported from the chromosphere without any cool seed in the corona. Our simulations not only suggest some condensation mechanisms of the hot plasma, but also show that cool material of whatever origin can survive in 

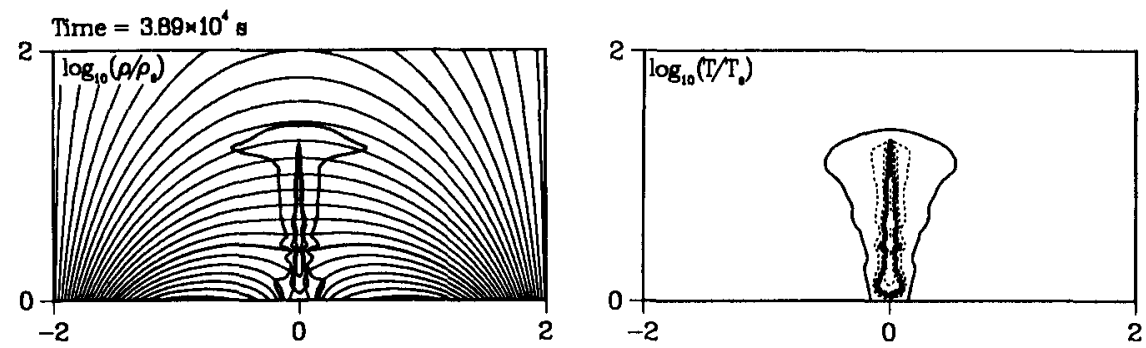

Figure 3. Case C: Formation of a prominence between two bipolar arcades. The density $\left(\log \left(\rho / \rho_{0}\right) \geq 0\right)$ contours are shown in the left panel superimposed on the magnetic field lines. In the right panel, the temperature $\left(\log \left(T / T_{0}\right)\right)$ contours are plotted.

the corona under certain conditions of the magnetic fields.

Besides the field geometries shown in this paper, Malville (in a discussion reported by Anzer 1979) proposed another field geometry as a plausible model of prominence magnetic fields. In Malville's model, a magnetic island is wrapped by Kippenhahn-Schlüter type field lines. In relation to this, the simulation study by Cheng and Choe (1998, these proceedings) has shown that Malville's configuration can be realized by magnetic reconnection in a current sheet whose lower tip lies above the bottom boundary.

Acknowledgments. This work was supported by the NSF grant ATM9696232 and the U. S. Department of Energy Contract DE-AC02-76-CHO3073. The authors would like to thank S. F. Martin and L. C. Lee for helpful discussions.

\section{References}

Anzer, U. 1979, in Physics of Solar Prominences, E. Jensen, P. Maltby and F. Q. Orrall, Institute of Theoretical Astrophysics, Blindern:Oslo, p. 322

Choe, G. S. 1995, Ph.D. Thesis, University of Alaska: Fairbanks

Choe, G. S. and Lee, L. C. 1992, Solar Phys., 138, 291

Martin, S. F. 1990, in Dynamics of Quiescent Prominences, (eds.) V. Ruždjak and E. Tandberg-Hanssen, Springer-Verlag:Berlin, p. 1

Rompolt, B. and Bogdan, T. 1986, Coronal and Prominence Plasmas, (ed.) A. Poland, NASA CP 2442, Washington, D.C., p. 81 